\title{
Aportes a la arqueología reduccional. El caso de Nuestra Señora de Ibitiracuá (Concepción de la Sierra, Misiones, Argentina)
}

\section{Amanda E. Ocampo*, Alejandro Richard"*, Yamila} Sastre ${ }^{* * * *}$ y Soledad Candelario ${ }^{* * * * *}$
Recibido:

21 de febrero de 2018

Aceptado:

6 de septiembre de 2018

\section{Resumen}

A partir de los primeros trabajos arqueológicos que se vienen realizando en el casco histórico de la localidad de Concepción de la Sierra (provincia de Misiones, Argentina), antiguo emplazamiento de la reducción jesuítica-guaraní Nuestra Señora de Ibitiracuá, y el análisis preliminar de los materiales recuperados en distintos sondeos, se discuten los datos obtenidos en relación a antecedentes regionales para momentos pre y posthispánicos. Se plantea el análisis de los atributos formales de las evidencias recuperadas en estas primeras intervenciones con el objetivo de identificar las características presentes en los materiales que se preservaron bajo la superficie, a pesar del alto dinamismo y disturbación del contexto arqueológico. Los resultados hasta ahora obtenidos avalan por un lado, la periodicidad de diferentes momentos históricos y por otro, la continuidad en determinados estilos cerámicos que se observa para tiempos prehispánicos. Por último, se discute el alto grado de reutilización espacial y material, como así también el rol de nuestra disciplina en torno a un patrimonio vivo y dinámico amenazado por la creciente urbanización.

\section{Contributions to reductional archaeology. The case of Nuestra} Señora de Ibitiracuá (Concepción de la Sierra, Misiones, Argentina)

\footnotetext{
Abstract

In this paper, we discuss the data obtained from archaeological studies in Concepción de la Sierra historical center (Misiones, Argentina), the former location of Nuestra Señora de Ibitiracuá Jesuit-Guarani reduction. We present the preliminary analysis

* Secretaría de Investigación y Postgrado, Facultad de Humanidades y Ciencias Sociales. Universidad Nacional de Misiones (UNaM) - CONICET. Tucumán 1632 (CP 3300). Posadas, Misiones, Argentina. E-mail: amiocampo25@gmail.com

** Centro de Arqueología Urbana (CAU), Instituto de Arte Americano (IAA), Facultad de Arquitectura, Diseño y Urbanismo, Universidad de Buenos Aires (UBA). Intendente Güiraldes 216o, Pabellón 3, $4^{\circ}$ piso, Ciudad Universitaria (CP C1428EGA) Ciudad Autónoma de Buenos Aires, Argentina. E-mail: ale_rich37@hotmail.com

***: Instituto de Arqueología y Museo, Facultad de Ciencias Naturales e Instituto Miguel Lillo, Universidad Nacional de Tucumán (UNT) / Museo de Ciencias Naturales y Antropológicas “Prof. A. Serrano”, Gobierno de Entre Ríos - CONICET. San Martín 1545 (CP T400oCWE), San Miguel de Tucumán, Tucumán, Argentina. E-mail: yamilasastre@hotmail.com.ar ***** Instituto Superior de Estudios Sociales (ISES), Facultad de Ciencias Naturales e Instituto Miguel Lillo, Universidad Nacional de Tucumán (UNT) - CONICET. San Martín 1545 (CP T400oCWE), San Miguel de Tucumán, Tucumán, Argentina. E-mail: soledadcandelario@gmail.com
}

Palabras clave

Arqueología histórica Materialidad

Reducción

Sitios

Reutilización 
Keywords Historical archaeology Materiality Reduction Sites Reuse

of the material evidence recovered from several test pits, and compare the results with previous regional data from the pre- and post- Hispanic periods. We analyze the formal attributes of the recovered material in order to identify the features preserved on sub-surface materials, in spite of the archaeological context high dynamism and disturbance. So far, the evidence supports the periodicity of the different historical periods and the continuity of some pre-Hispanic ceramic styles. Finally, we address the high degree of material and spatial reuse in this and other reductions in Misiones Province. We also argue for our discipline role in protecting living, dynamic heritage sites increasingly threatened by urbanization.

\section{Introducción}

Los espacios reduccionales jesuíticos-guaraníes (1610-1768) que se establecieron en la región misionera - sureste de Paraguay, sur de Brasil, nordeste de Argentina y norte de Uruguay- constituyen uno de los patrimonios históricos más importantes de la actual provincia de Misiones. Uno de ellos es el actual municipio de Concepción de la Sierra, centro de interés de la presente investigación (Figura 1).

El actual poblado se encuentra emplazado sobre el trazado urbano jesuítico del siglo XVII. La materialidad asociada a este último se compone de diversos materiales: fragmentos de tejas, lozas y cerámicas asociadas a distintos momentos históricos, distinguiéndose además materiales constructivos reutilizados como: areniscas e itacurú canteadas, columnas, dinteles, entre otros.

El objetivo del presente trabajo es proponer una línea de estudio que analice la continuidad histórica y la reutilización espacial y material del sitio, a partir de dicha materialidad arqueológica (Ocampo, 2015; Ocampo y Núñez, 2015; Ocampo y Richard, 2016; Ocampo, Sastre y Lallami, 2015), de manera que permita una aproximación preliminar en la arqueología reduccional y de post-expulsión jesuítica del sitio. Al respecto, nos propusimos como objetivo particular, identificar las características presentes en los materiales arqueológicos que se preservan bajo la superficie, a pesar del alto dinamismo y disturbación propios del contexto descripto a partir de un análisis de los atributos formales del conjunto cerámico recuperado (Ocampo y Richard, 2016).

\section{Metodología de trabajo}

A partir de la información obtenida de la documentación histórica, el análisis de esquemas urbanísticos de la antigua reducción y su superposición con el plano del pueblo actual, se pudo determinar el emplazamiento de las principales edificaciones (capilla, cabildo, viviendas, piletas, entre otros) presentes en el antiguo poblado (Ocampo y Richard, 2016).

Las prospecciones llevadas a cabo en el casco histórico de Concepción de la Sierra (CS) fueron de tipo pedestre, arbitrarias y sin recolección superficial. Se realizó un recorrido a pie por transectas definidas por la disposición del trazado de las calles, pudimos identificar puntos específicos en el espacio para efectuar sondeos arqueológicos. Por su parte, los permisos otorgados por los propietarios de los terrenos privados permitieron una inspección ocular en los mismos y así excavar un mayor número de sondeos.

Los sondeos realizados fueron de $1 \mathrm{x} 1 \mathrm{~m}$ y se efectuaron en los sitios Concep 1, 2, 3, 4 y 5 (Figura 2). La materialidad recuperada fue registrada y embalada por separado, 


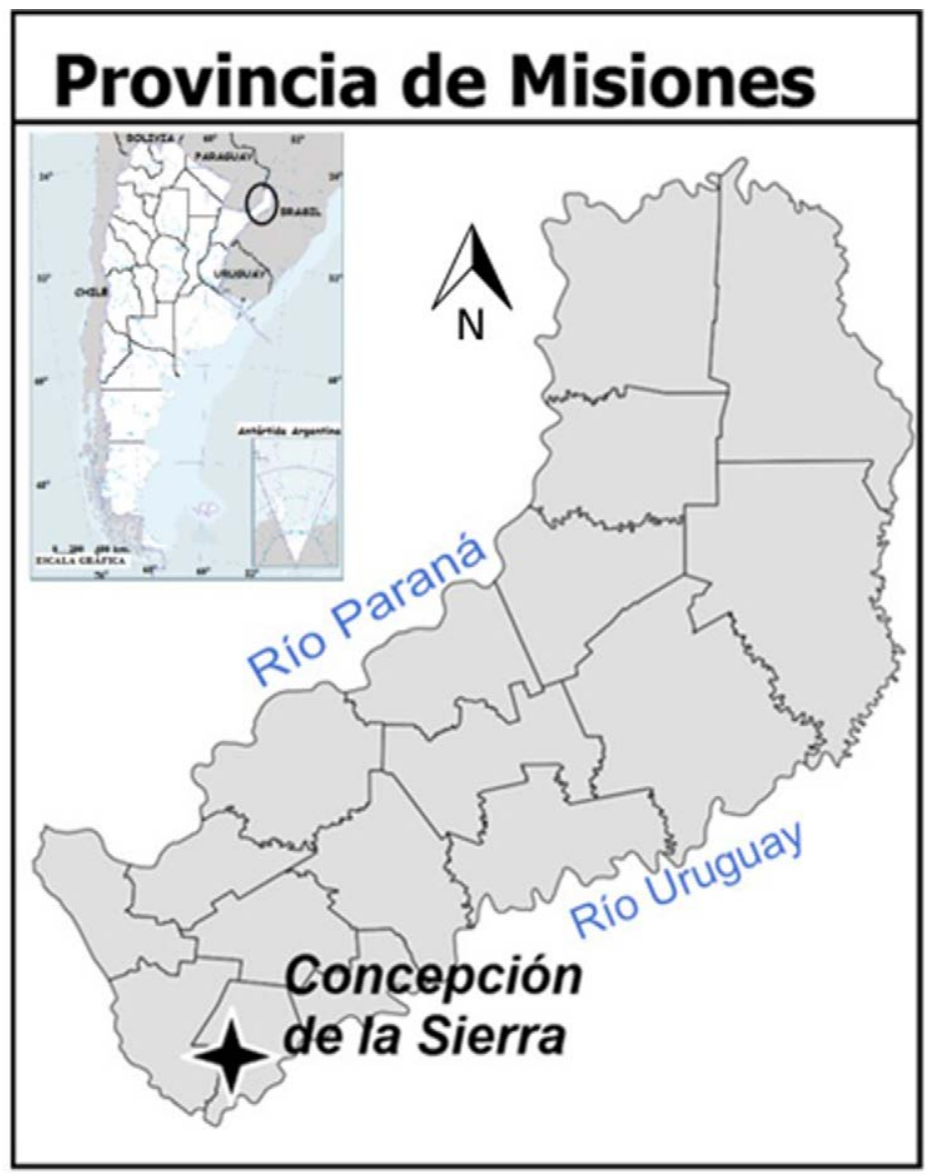

Figura 1. Ubicación geográfica de Concepción de La Sierra, provincia de Misiones.

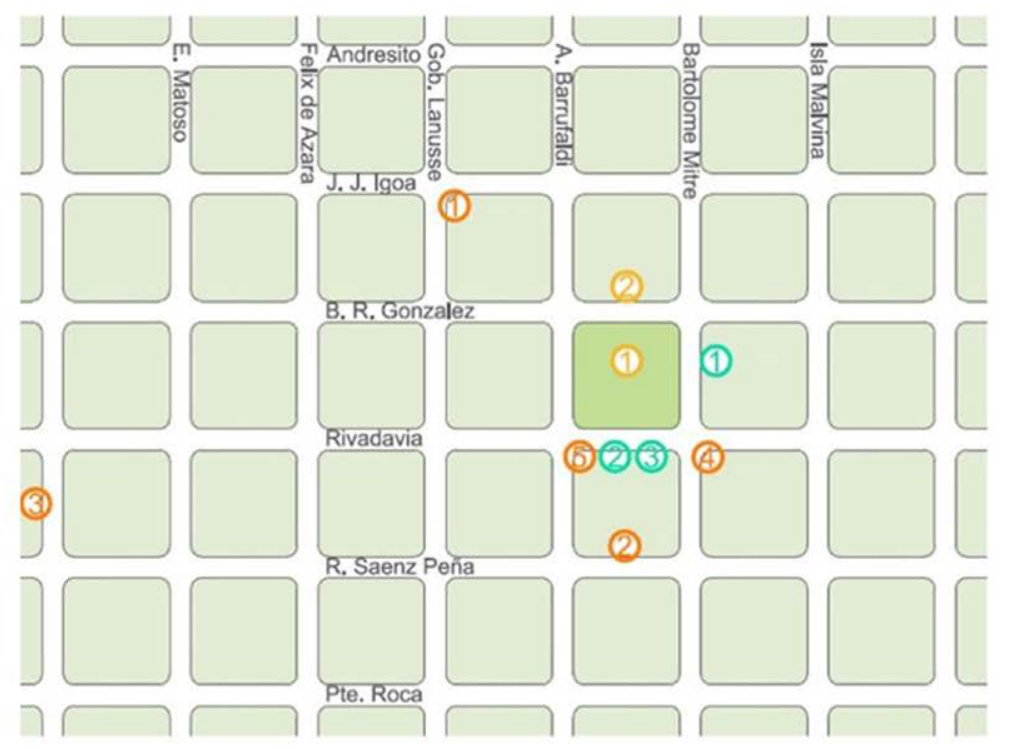

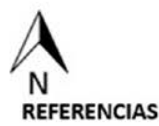

\section{REFERENCIAS}

LUGARES PRINCIPALES DEL PUEBLO

1- Plaza principal

2. Casa de la Cultura

SITIOS ARQUEOLÓGICOS RELEVADOS

1- Sitio CONCEP 1. Propiedad privada

2- Sitio CONCEP 2. Propiedad privada

3- Sitio CONCEP 3. Piletas jesuíticas. Chacra privada

4- Sitio CONCEP 4. Ministerio de Agricultura

5- Sitio CONCEP 5. Terreno vacío con propietario ESPACIOS REUTIUZADOS RELEVADOS

1- Antiguo templo jesuítico- actual iglesia

2-Antiguo cabildo- actual casa Márquez

3- Cotiguazú- actual vivienda particular 
diferenciando entre tejas, ladrillos, loza, vidrio, metal, restos óseos y cerámica. Posteriormente se realizaron análisis macroscópicos y se completaron fichas diseñadas para cada material.

El material recuperado fue lavado con cepillos de cerdas finas y agua, con el fin de remover el sedimento adherido permitiendo la observación de sus características formales. Posteriormente, se rotuló cada fragmento consignando año de excavación, sitio, sondeo y nivel de procedencia.

\section{Resultados}

A continuación, presentamos un plano de los sondeos realizados, con el objetivo de identificar probables relaciones existentes entre los materiales recuperados y la espacialidad ligada a la antigua reducción jesuítica-guaraní. Estos se realizaron en cinco sitios definidos: Concep 1, 2, 3, 4 y 5 (ver Figura 2). Si bien cada uno de ellos presenta diferencias en cuanto a las características de emplazamiento y material arqueológico recuperado, muestran en común la reutilización de los espacios (Ocampo et al., 2015).

\section{Materiales recuperados}

A continuación, presentamos un análisis descriptivo de los materiales recuperados que fueron estudiados en laboratorio.

\section{Cerámica}

Se analizaron un total de 17 fragmentos: tres provenientes del sitio Concep 1, dos del sitio Concep 2 y 12 del sitio Concep 5 (Figura 3). De este último sitio procede la mayor cantidad de alfarería recuperada hasta el momento. La muestra cerámica en general presenta un buen estado de conservación, exceptuando algunos fragmentos (la mayoría proveniente de Concep 5) cuyas superficies externas e internas se encuentran erodadas en distintos niveles. Con respecto a las dimensiones de los tiestos, el tamaño varía entre los 3 y $6 \mathrm{~cm}$, seguido por los menores a $3 \mathrm{~cm}$ y en menor cantidad entre los 6 y $12 \mathrm{~cm}$.

En estos tres sitios se registró la presencia de cerámica con superficie externa corrugada correspondientes a fragmentos de borde. A su vez, el espesor máximo está entre los $9 \mathrm{y}$ $12 \mathrm{~cm}$, cocción reductora u oxidante incompleta, tiestos molidos y arena cuarzosa en sus pastas. En lo que se refiere a huellas de uso, un fragmento procedente del sitio Concep 5 tiene restos de hollín. Por otro lado, en este sitio también se recuperaron cuatro tiestos con engobe y superficie externa bruñida, de los cuales tres remontan constituyendo un borde con una acanaladura longitudinal al mismo. Este material tiene un espesor máximo de $6 \mathrm{~cm}$, cocción oxidante incompleta y arena cuarzosa en sus pastas. Estas características compartidas entre el borde remontado y el fragmento de cuerpo en lo que respecta al tipo de antiplástico, espesor de las paredes, tratamiento de la superficie y atmósfera de cocción nos llevan a pensar que habrían pertenecido a una misma pieza.

Por último, para completar con la descripción de la muestra analizada, debemos mencionar que en el conjunto de Concep 1 se registraron dos fragmentos con distintos atributos: uno alisado con antiplásticos de arena cuarzosa y de cocción oxidante incompleta y otro con engobe y alisado, con una pasta donde no se observó a simple vista material antiplástico y de cocción reductora. En Concep 2 también se recuperó un tiesto que evidencia restos de pintura roja en la superficie externa, cocción oxidante incompleta y una pasta con arena cuarzosa, material orgánico y posibles restos de conchillas. Finalmente, completando con la descripción del conjunto de Concep 5, en los casos en que los fragmentos no 


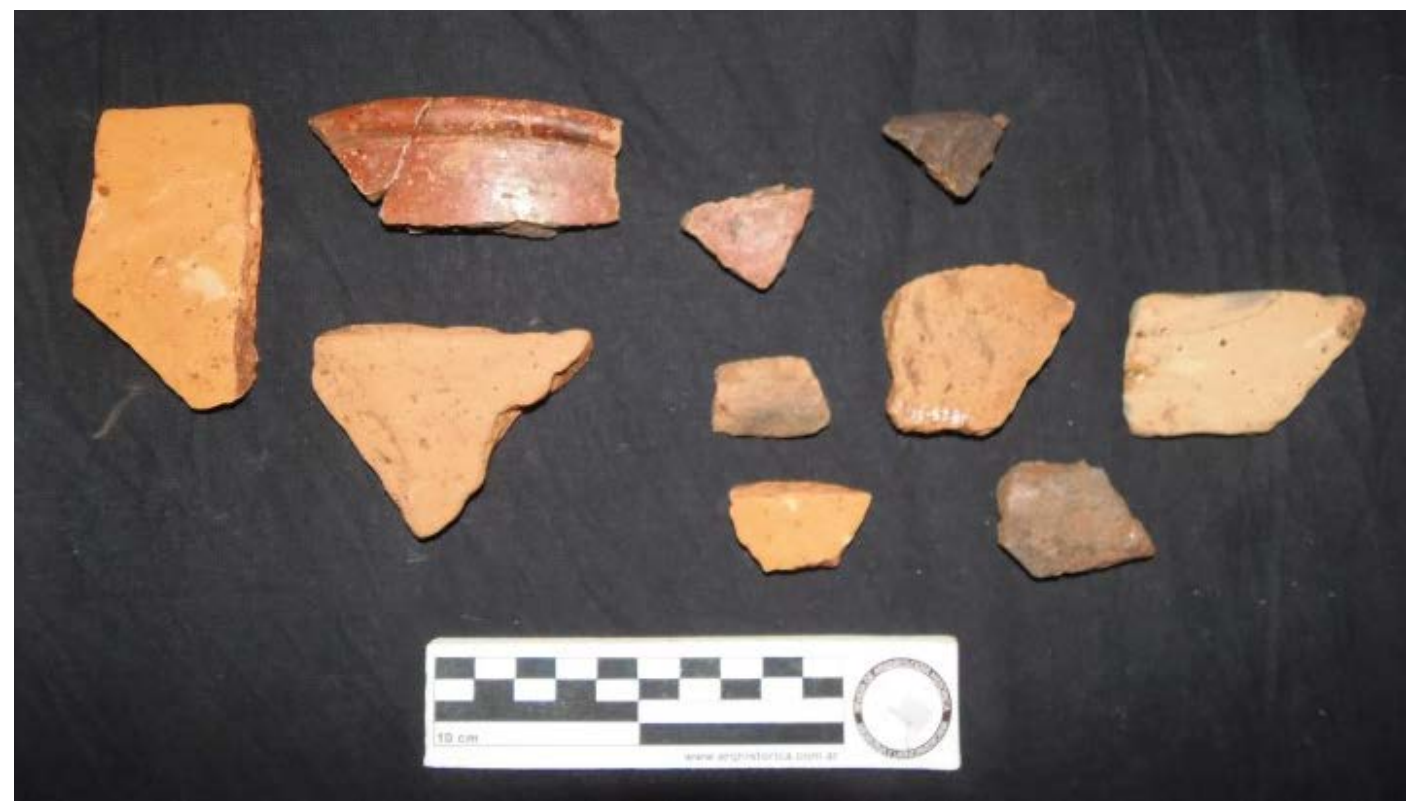

Figura 3. Fragmentos cerámicos analizados.

presentaban sus superficies externas erodadas, fue posible registrar tiestos con engobe o alisados. El tipo de cocción y los tipos de antiplásticos (arena cuarzosa, tiesto molido, arena cuarzosa y carbón, arena cuarzosa y tiesto molido, arena cuarzosa y hueso molido) evidencian que la cerámica de este conjunto es variada.

\section{Tejas}

La muestra analizada está compuesta por 178 fragmentos de tejas: 18 provenientes del sitio Concep 1, 127 del sitio Concep 2, 31 del sitio Concep 3 y dos del sitio Concep 4 (Figura 4). El mayor número de los fragmentos analizados proviene de los sondeos 1 y 4 del sitio Concep 2, donde se recuperaron 127 fragmentos (71,7\% del total). En ninguno de los sitios excavados se recuperaron tejas completas. De los fragmentos analizados, un $35,3 \%$ presenta dimensiones menores a $5 \mathrm{~cm}$ de lado, mientras que un $52,9 \%$ posee entre 5 y $10 \mathrm{~cm}$ y sólo el $11,7 \%$ del total tiene dimensiones superiores a los $10 \mathrm{~cm}$. El lote más fragmentado proviene del sitio Concep 1, donde se observan dimensiones inferiores a los $5 \mathrm{~cm}$ en un $47,1 \%$ de la muestra. Los fragmentos que presentan erosión componen el 35,2\% del total, estando representados en mayor medida en Concep 2 (50,4\%). El tamaño de los restos de tejas posiblemente se debe a procesos tafonómicos los cuales alteran los conjuntos de materiales, sumando además el contexto de alto contenido de humedad, suelos arcillosos y remoción del mismo por obras de construcción actuales.

En el 64\% de la muestra se observaron escorias metálicas presentes en pasta. Estas concentraciones semiesféricas, con tamaños de entre 1 y $2 \mathrm{~mm}$, se encuentran en los fragmentos de tejas de todos los sitios estudiados. Su cocción es oxidante, mientras que en un $11,1 \%$ esta se desarrolla de forma incompleta.

\section{Lozas, gres y semiporcelana}

El total de la muestra está constituido por 18 fragmentos cerámicos correspondientes al sitio Concep 2. La mayoría procede del sondeo $\mathrm{n}^{\circ} 4$, conformado por 13 fragmentos y, en menor 


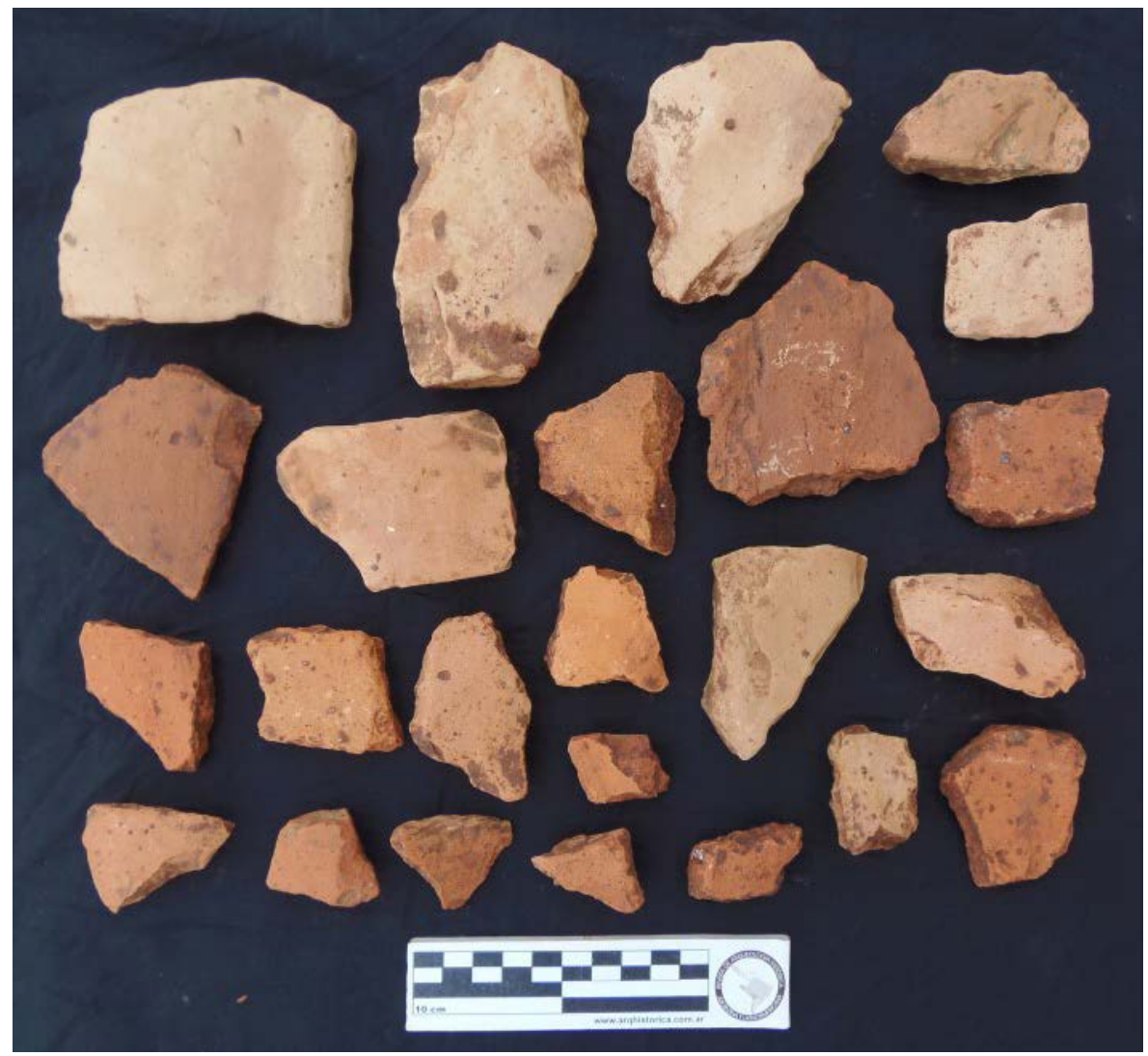

Figura 4. Fragmentos de tejas recuperados.

medida, se destacan tres fragmentos del sondeo $\mathrm{n}^{\circ} 5 \mathrm{y}$ dos fragmentos del sondeo $\mathrm{n}^{\circ} 2$. El estado de conservación de la muestra es fragmentario, con algunos defectos en los vidriados de los mismos, principalmente en las lozas a las que hacemos referencia más adelante.

Los fragmentos se clasificaron de acuerdo al material de manufactura, siguiendo a Schávelzon (2001) y Frazzi (2009). De esta manera, se obtuvieron los siguientes grupos cerámicos: lozas, gres y semiporcelana. Un total de 15 fragmentos de loza, dos fragmentos de gres y un fragmento de semiporcelana.

Las lozas analizadas componen un total de 15 fragmentos: 12 de ellas provienen del sondeo $\mathrm{n}^{\circ} 4$, dos fragmentos del sondeo $\mathrm{n}^{\circ} 5 \mathrm{y}$ un fragmento del sondeo $\mathrm{n}^{\circ} 2$. Poseen dimensiones de entre 10 y $60 \mathrm{~mm}$ de lado y espesores de entre 4,5 y $7 \mathrm{~mm}$ (Figura 5). A partir de la observación de las cubiertas vítreas de los diferentes fragmentos, podemos determinar que sólo en un caso, en el que el fragmento corresponde a un asa o manija de recipiente, se distingue un tinte azulado, propio de la loza Pearlware, a la que se le incorporaba cobalto durante el proceso de manufacturación (Schávelzon, 1991, 2001). Los restantes fragmentos presentan una cubierta vítrea transparente, propia de las lozas Whiteware (Frazzi, 2009; Schávelzon, 1991).

Los defectos del vidriado observados en la muestra son el punteado, el craquelado y el nodulizado. Estos se deben a las partículas de polvo al momento del horneado, a excesivas temperaturas del horno y al exceso de esmalte en la pieza cerámica respectivamente (Frazzi, 2009).

Respecto a la decoración de las lozas, 13 fragmentos corresponden al tipo blanco liso, mientras que sólo dos presentan decoración del tipo borde decorado (Schávelzon, 


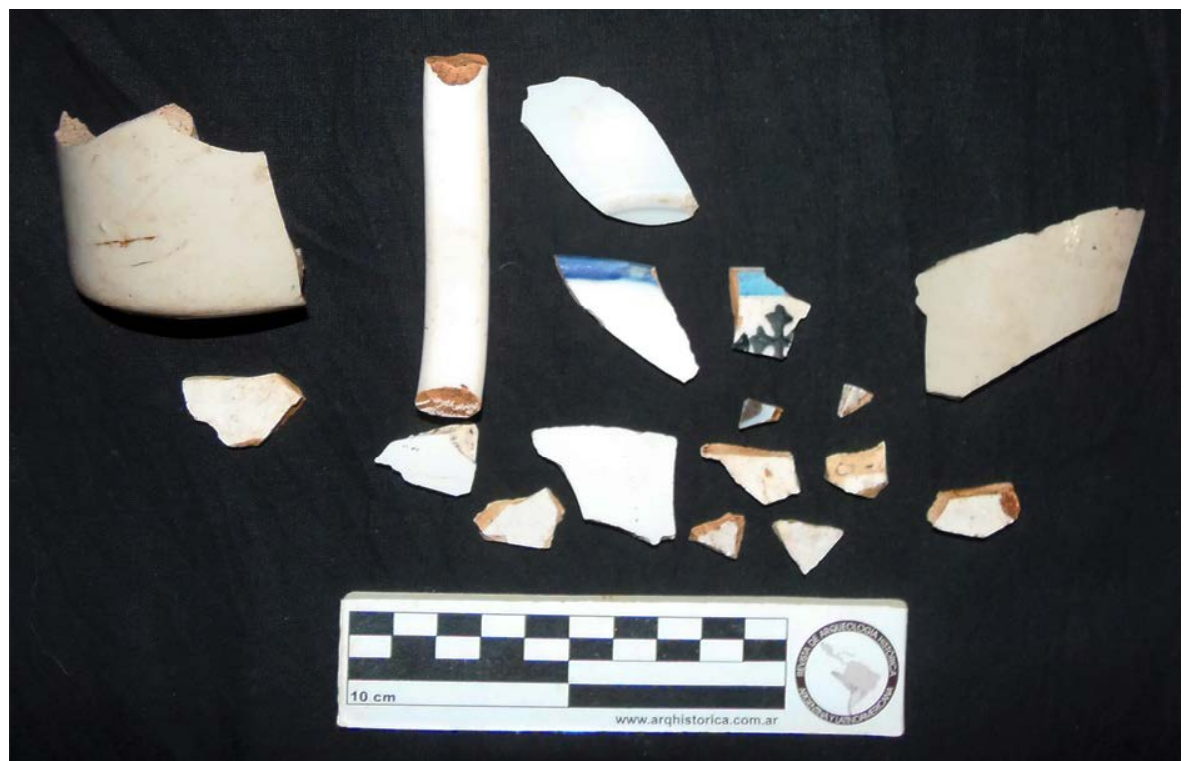

Figura 5. A) Fragmento de base de botella cilíndrica de gres y manija de loza Pearlware; B) fragmentos del tipo borde decorado; C) fragmento de cuerpo de botella cilíndrica de gres y fragmentos de lozas Whiteware.

2001). Éstos últimos presentan motivo de borde decorado en azul, aunque uno de ellos se diferencia por poseer la misma técnica en el borde interior y en la cara externa un motivo similar a una cruz en color negro.

Por otra parte, se analizaron dos fragmentos de gres del total de la muestra $(\mathrm{n}=18)$. Uno de ellos proviene del sondeo $\mathrm{n}^{\circ} 5$ y mide aproximadamente entre 30 y $60 \mathrm{~mm}$, mientras que el otro fragmento fue recuperado del sondeo $\mathrm{n}^{\circ} 2$ con dimensiones mayores a $60 \mathrm{~mm}$. Los espesores varían entre 80 y $110 \mathrm{~mm}$. Los fragmentos tienen color crema, destacándose un vidriado brillante externo y marcas de torno en su interior. Morfológicamente forman parte de la base y del cuerpo de una botella cilíndrica. Por las características generales destacadas los fragmentos corresponderían a una botella de gres de cerveza (Schávelzon, 1991, 2001).

Respecto al análisis macroscópico de la pasta observamos pequeños puntos oscuros en la matriz del gres, que pueden estar relacionados a la de materia orgánica obtenida durante la cochura de la pieza, aunque éste indicador hasta ahora sólo se describió para las lozas (Frazzi, 2009).

Además, se pudo distinguir de la muestra total analizada, un fragmento de un material diferente al que denominamos semiporcelana, recuperada del sondeo $\mathrm{n}^{\circ} 4$, con dimensiones entre 3 y $6 \mathrm{~mm}$ y un espesor de $4,5 \mathrm{~mm}$. El material de manufactura consiste en una pasta blanca, más dura y menos porosa que una loza, con vidriado transparente y de acabado brillante. Por las características que y su espesor sugerimos que se trata de un fragmento de "porcelana blanda" (Frazzi, 2009).

\section{Discusión. Integrando datos}

A partir de los sondeos realizados en múltiples puntos del casco histórico de Concepción de la Sierra y un estudio a nivel macroscópico de los materiales recuperados, establecemos las primeras interpretaciones de los datos teniendo en cuenta los antecedentes regionales. 
En lo que respecta a la alfarería con superficie externa corrugada, esta técnica fue utilizada frecuentemente en los sitios arqueológicos asociados a grupos guaraníes ( $\mathrm{La}$ Salvia y Brochado, 1989; Loponte y Acosta, 2013; Neuman, 2009; Prous y Jacome, 2011; Schmitz, 2008, entre otros).

En nuestro caso en particular, observamos que la técnica del corrugado y la adición de tiestos molidos fueron también atributos registrados en la alfarería guaraní de la ocupación reduccional de Concepción.

Halberstadt (2013), en un análisis de los tiestos procedentes del sistema pluvial de la Reducción Jesuita de Santa Ana (Misiones), menciona la presencia de un conjunto cerámico pintado con engobe rojo, bruñido y, en algunos fragmentos, la existencia de líneas rectas o círculos concéntricos incisos sobre la superficie externa. Tanto la pintura monocroma roja, como el tratamiento de superficie mencionado, también fueron observados en fragmentos del conjunto de Concep 5.

En general, los restos cerámicos recuperados presentan características similares a los registrados en otros sitios reduccionales tales como en San Ignacio Miní o Santa Ana (Poujade, Roca y Salvatelli, 2016). En cuanto a los otros materiales cerámicos analizados, se pudo inferir que los restos de lozas, gres y semiporcelana corresponderían al período de ocupación que se extiende desde fines del siglo XVIII, representado por la loza Pearlware (1780-1840) y a lo largo del siglo XIX y XX, con mayor proporción de la muestra en los dos últimos siglos, con la presencia de lozas Whiteware (1809 - hasta la actualidad). La gran mayoría de los fragmentos hallados del tipo blanco liso se asocia a un material fabricado industrialmente y dirigido al consumo de la clase media (Schávelzon, 1991).

La loza decorada más significativa en la primera mitad del siglo XIX es la de borde decorado color azul (Schávelzon, 1991). Se trata de una técnica que se aplica en los tres tipos de lozas blancas y que en el caso analizado corresponden al esmalte trasparente típico de las lozas Whiteware. El gres, escasamente representado, corresponde a ejemplares del siglo XIX (Schávelzon, 2001).

Los fragmentos de tejas, los cuales se ven representados en todos los sitios expuestos en el presente trabajo, poseen a su vez una alta representatividad en múltiples sitios reduccionales excavados de la región (Poujade, 2000; Poujade et al., 2016; Poujade, Austral, Rochietti y De Grandis, 2006; entre otros). Consideramos por ende necesario aportar al estudio sistemático de esta materialidad desde sus características formales, a fin de indagar en los procesos productivos y las cronologías relativas asociadas a estos.

En cuanto a la muestra analizada, destacamos la presencia de escorias metálicas en pasta y la predominancia de la cocción oxidante durante el proceso de elaboración. En su contexto arqueológico, el material estudiado se encontró asociado tanto a cerámica guaraní como a fragmentos de loza. Esto puede deberse a la persistencia de su utilización a lo largo del tiempo, o meramente a procesos postdepositacionales que dieron origen a su alta fragmentación y representación a lo largo de toda la secuencia estratigráfica. En lo que se refiere a las mencionadas escorias, consideramos que futuros estudios que aborden la litología y los depósitos de arcillas locales permitirán identificar el origen de la materia prima utilizada para su elaboración.

A partir de los análisis expuestos, se observa una continuidad de ocupación manifestada de materiales asociados a distintos momentos de ocupación, desde el período reduccional (siglos XVII y XVIII) hasta los siglos XIX y XX. 


\section{Consideraciones finales}

Los trabajos arqueológicos realizados con el objetivo de abordar el estudio de las ocupaciones jesuítico-guaraníes en la antigua Reducción de Nuestra Señora del Ibitiracuá, en el pueblo de Concepción de la Sierra, permitieron definir distintos sitios considerándolos parte integral de una reducción en contextos urbanos actuales. Las intervenciones efectuadas llevaron a recuperar y analizar una materialidad arqueológica inserta en un contexto de reocupación espacial, las que a su vez constituyen el estudio del patrimonio arqueológico reduccional en continua transformación.

Los resultados obtenidos hasta el momento nos llevan a reconocer, por un lado, diferentes períodos o momentos históricos, y por otro, una continuidad entre los estilos cerámicos prehispánicos y reduccionales, probablemente reproducidos inclusive ante la influencia de estilos europeos (Brochado, Monticelli y Neumman, 1990). Si bien este trabajo reproduce los primeros registros de nuestra investigación, desde lo observado en materiales tales como la cerámica, podemos inferir a partir de las huellas de uso que la alfarería formó parte de piezas expuestas al fuego y que, morfológicamente podrían corresponder a ollas, cazuelas, tinajas o platos (Pérez, 2016), es decir, recipientes vinculados con la preparación de alimentos.

En cuanto a los materiales de loza, gres y semiporcelana podemos destacar diferentes usos a partir de la reconstrucción de las formas más habituales o comunes halladas en fragmentos por Schávelzon (2001), entre estas sobresalen las lozas utilizadas como vajilla de mesa en: platos, vasos, fuentes o tazas, así también otras formas como el asa de loza Pearlware puede reflejar un uso no solo como vajilla sino también ornamental, como ser una manija de alguna fuente o florero, al tiempo que el gres es mencionado principalmente como envase de bebida. Cabe destacar que existen formas habituales para cada tipo cerámico asociando los mismos directamente a determinado uso, sin embargo, este criterio no es exclusivo en la medida en que consideremos que las formas de uso cambian de acuerdo con sus usuarios (Schávelzon, 2000).

Destacamos además el hecho de que los trabajos arqueológicos en la provincia, en contextos urbanos, sirven como actividades de rescate del registro arqueológico e histórico con el fin de preservar y poner en valor al acervo cultural de la región (Ocampo et al., 2015).

Si tenemos en cuenta que todo el espacio que representa el casco histórico fue parte de la antigua Reducción, se deduce que la planificación urbana actual es un espacio reutilizado. Esta característica particular de reutilización se define como un proceso de reaprovechamiento de un lugar en cuestión. En este caso de estudio, hay un espacio de planificación urbana típica de una antigua reducción jesuítica-guaraní, la cual atraviesa un proceso de reciclaje en un período posterior y el espacio está desarrollando un nuevo modelo urbano, a los fines del nuevo pueblo, con funciones similares a su antigua ocupación. La reutilización de estas planificaciones urbanas de la Compañía de Jesús actualmente se encuentra en directa relación con el pueblo y sus actividades, generando una manifestación de continuidad histórica con su pasado. Los procesos de reutilización implican la articulación de diferentes momentos, formando parte de la evolución urbana y social de la historia del lugar (Ocampo y Núñez, 2015).

Desde un enfoque estrictamente arqueológico y metodológico, el espacio estudiado se presenta como un factor de obstrusividad, es decir, que aumenta la probabilidad de que materiales arqueológicos depositados en la superficie terrestre puedan ser descubiertos por un/a operador/a (Arrigoni, 2014).

Como futuras líneas de investigación consideramos la necesidad de realizar trabajos de archivo con fuentes documentales, las que permitirán articular la información 
resultante con aquella obtenida a partir de los análisis de laboratorio. Finalmente argumentamos que el carácter de este informe preliminar, que muestra el desarrollo de un proyecto de investigación en sus primeras etapas, lo que busca es establecer posibles líneas de estudio a futuro y concretas metodologías de trabajo en sitios que fueron antiguas reducciones de jesuitas y guaraníes que actualmente se encuentran en contextos de transformación urbana.

\section{Agradecimientos}

La investigación en curso se realiza gracias a las siguientes instituciones: el CONICET y la Facultad de Humanidades y Ciencias Sociales de la Universidad Nacional de Misiones. También agradecemos al municipio de Concepción de la Sierra por el acompañamiento de siempre y su hospitalidad hacia nosotros y a los/as evaluadores/as externos/as que se tomaron el trabajo de revisar minuciosamente este artículo. Nuestros sinceros agradecimientos. 


\section{Q Referencias citadas}

" Arrigoni, G. L. (2014). Evaluación del impacto arqueológico “Construcción de gasoducto CT-2 A PCG CD-1". Estudio de impacto ambiental arqueológico. Hidroar S. A. Servicios Hidrogeológicos y ambientales, Comodoro Rivadavia, Chubut. Manuscrito inédito.

》 Brochado, J.P., Monticelli, G. y Neumann, E. (1990). Analogia etnográfica na reconstrução das vasilhas Guarani arqueológica. Veritas, 35, 727-743.

» Frazzi, P. (2009). Conservación y Restauración de Lozas de excavaciones arqueológicas de Buenos Aires y su periferia. (Tesis de Licenciatura inédita), Instituto Universitario Nacional de Arte, Argentina.

" Halberstadt, L. (2013). El arte misional en pintura, escultura y cerámica: una perspectiva artística e histórico antropológica. Trabajo presentado en las I Jornadas Interdisciplinarias de Jóvenes Investigadores en Ciencias Sociales. IDAES/UNSAM. San Martín, Argentina.

» La Salvia, F. y Brochado, J. (1989). Cerâmica Guarani. Porto Alegre: Posenato Arte \& Cultura.

"Loponte, D. y Acosta, A. (2013). La construcción de la unidad arqueológica Guaraní en el extremo meridional de su distribución geográfica. Cuadernos del Instituto Nacional de Antropología y Pensamiento Latinoamericano (Series Especiales), 1(4), 193-235.

" Neuman, E. (2009). Questo es metodológicas para a análise das marcas de uso na cerâmica Guaraní Arqueológica. Revista do Museu de Arqueologia e Etnologia, 8, 225-230.

"Ocampo, A. E. (2015). Entre la ciudad y la selva: una reducción jesuítica guaraní en contextos de transformación urbana. Concepción de la Sierra, Misiones, Argentina. Trabajo presentado en el VI Encuentro de Discusión Arqueológica del Nordeste Argentino. Gualeguaychú, Argentina.

"Ocampo, A. E. y Richard, A. (2016). Reutilización espacial en las misiones jesuíticaguaraní. El caso del pueblo de Concepción de la Sierra (Misiones, Argentina). Revista del Museo de Antropología de Entre Ríos, 2(1), 88-99.

》 Ocampo, A. E y Núñez, M. (2015). La importancia de los pueblos históricos de Misiones. Diálogos entre arqueología y turismo cultural en Concepción de la Sierra. Trabajo presentado en las Jornadas de Investigadores 2015. "Fronteras y liminaridades. Espacios de diálogo, confrontación y descubrimiento". Posadas, Argentina.

"Ocampo, A. E., Sastre, Y. y Lallami, C. (2015). Arqueología Histórica en contextos urbanos. El caso de la reducción jesuítica-guaraní “Nuestra Señora de la Concepción" (Concepción de la Sierra, Misiones, Argentina). Trabajo presentado en el VI Congreso Nacional de Arqueología Histórica Argentina. Mendoza, Argentina.

»Pérez, M. (2016). Cerámica arqueológica del Delta de Paraná. (Tesis Doctoral inédita), Universidad de Buenos Aires, Argentina.

》Poujade, R. (2000). Arqueología de las Reducciones jesuíticas de Guaraníes de Misiones. Trabajo presentado en el I Congreso de Arqueología Histórica Argentina. Buenos Aires, Argentina.

"Poujade, R. A., Austral, A., Rochietti, A. M. y De Grandis, N. (2006). Arqueología de Santa Ana, Misión Jesuita (Misiones - Argentina). Trabajo presentado en el III Congreso Nacional de Arqueología Histórica. Escuela de Antropología. Facultad de Humanidades y Artes, Universidad Nacional de Rosario. Santa Fe, Argentina. 
» Poujade, R., Roca, M., y Salvatelli, L. (2016). Intervenciones arqueológicas en cuatro conjuntos Patrimonio Mundial. Reducciones de Santa Ana, San Ignacio Mini, Santa María La Mayor y Nuestra Señora de Loreto (Misiones, Argentina). En L. M. Calvo y G. Cocco (Comps.), Primeros asentamientos españoles y portugueses en la América central y meridional. Siglos XVI y XVII (pp. 339- 353). Santa Fe: Universidad Nacional del Litoral.

» Prous, A. y Jacome, C. (2011) El ser por el hacer: La socialización y la identidad expresadas por la cerámica tupi guarani. En M. C. Páez y G. A. de la Puente (Eds.), La Cerámica Arqueológica en la Materialización de la Sociedad. Transformaciones, Metáforas y Reproducción Social (pp. 45-58). Oxford: British Archaeological Report (BAR).

»Schávelzon, D. (1991). Arqueología Histórica de Buenos Aires. La cultura material porteña de los siglos XVIII y XIX. Buenos Aires: Ediciones Corregidor.

»Schávelzon, D. (2000). Historia del comer y del beber en Buenos Aires. Buenos Aires: Editorial Aguilar.

»Schávelzon, D. (2001). Catálogo de cerámicas históricas de Buenos Aires (siglos XVI-XX). Buenos Aires: Fundación para la investigación del Arte Argentino.

» Schmitz, P. A. (2008). Decoração plástica na Cerâmica da tradição Tupi guaraní. En A. Prous y T. Andrade Lima (Eds.), Os Ceramistas Tupi guaraní (Vol. 2, pp. 7-26). Brasilia: Instituto do Patrimônio Histórico e Artístico Nacional. 